Introduction: The use of immunotherapy in Mexico has been used since 2012 with ipilimumab and since 2015 with nivolumab and pembrolizumab, so it is a matter of necessity to know the experience of these drugs. Material and methods: An observational, descriptive, cross-sectional, and retrospective study was performed in Médica Sur Hospital, where with dossiers from 2012 to June 2018 patients with metastatic cancer who received immunotherapy with ipilimumab, nivolumab, and pembrolizum$a b$ for six months were evaluated, searching as principal outcomes the adverse effects of those drugs and as secondary outcomes the response to treatment.

Results: Seventy subjects fulfilled the inclusion criteria for the study, and 42 $(60 \%)$ were women with an average age of $60.73 \pm 13.64$ years (16-82 years). The pathologies that received immunotherapy were the following: melanoma and lung cancer. The most frequent clinical and laboratory adverse effects were as follows: fatigue - 32 (45.71\%), asthaenia - 30 (42\%), nausea $8(11.4 \%)$, diarrhoea -8 (11.4\%), and rash -7 (10\%). The worst adverse effects were respiratory and endocrinological: pneumonitis - 10 (14.28\%), hypothyroidism - 4 (5.71\%), hyperglycaemia - 1 (1.4\%), and hypophysitis $2(2.9 \%)$. With respect to treatment response: complete response $-8(11.4 \%)$, partial response $-11(15.71 \%)$, stable disease - $33(47.14 \%)$, and disease progression - 19 (27.14\%).

Conclusions: The most common adverse effects did not condition the suspension of treatment or increase in intra-hospital stay, but there were some adverse effects that actually had an impact on evolution, hospital stay, and mortality.

Key words: cancer, immunotherapy, ipilimumab, nivolumab, pembrolizumab.

Contemp Oncol (Pozn) 2019; 23 (4): 239-246 DOI: https://doi.org/10.5114/wo.2019.91534

\section{Cancer immunotherapy experience in the Integral Oncology Centre "Diana Laura Riojas de Colosio", Médica Sur Hospital}

\author{
Ricardo Fernández-Ferreira ${ }^{1}$, Daniel Motola-Kuba ${ }^{2}$, \\ lleana Mackinney-Novelo², José Manuel Ruiz-Morales², \\ María Eugenia Torres-Pérez ${ }^{1}$
}

${ }^{1}$ Internal Medicine Department, Médica Sur Hospital, Mexico City, Mexico ¿Oncology Medicine Department, Integral Oncology Centre “Diana Laura Riojas de Colosio", Médica Sur Hospital, Mexico City, Mexico

\section{Introduction}

The National Cancer Institute's definition of immunotherapy refers to any treatment that impulses or restores the capability of the immunological system to fight cancer, infections, or other diseases [1]. To be effective immunotherapy needs to increase the quality or quantity of immune active cells, expose additional tumoral protective antigens (i.e. propagation of the antigen) or inhibit the cancer-induced immunosuppressive mechanisms [2].

Immunology cancer treatment investigation has led to the discovery of regulation points between the interaction of antigen-presenting cells, cytotoxic T or immune-cells, and tumour cells. From these T-cell checkpoints, currently studied are cytotoxic T-lymphocyte antigen 4 (CTLA-4), programmed cell death protein 1 (PD-1), and one of its ligands (PD-L1). This knowledge has allowed the creation of monoclonal antibodies, which block the T-cell checkpoint signalling via CTLA-4 and PD-1 or PD-L1, thereby releasing the brakes on T-cell function and bolstering its cytotoxicity. These antibodies have demonstrated clinically important benefits in tumours like melanoma, lung cancer, renal cell cancer, colon cancer, lymphoma, and bladder cancer. However, there are multiple studies in which the utility of these antibodies is being proved, to determine their activity in other conditions [3, 4].

CTLA-4 was discovered in the early 1980s; it is a member of the immunoglobulin superfamily that acts as a negative immune system regulator and plays a key role in the inhibition of antitumour immunity [5]. PD-1 was discovered in 1992 for its high expression during apoptosis in a T-cell hybridoma model. It is expressed in activated $T$ and $B$ cells and thymocytes $[6,7]$. Two ligands have been described: PD-L1 and PD-L2 (also denominated B7-H1 and B7-DC, respectively), both of which are type 1 transmembrane glycoproteins with type IgC and IgV extracellular domains [8]. The expression of PD-L1, both in lymphoid tissues and non-lymphoid tissues, suggests that PD-1/PD-L1 pathway might modulate the immune responses in secondary lymphoid tissues as in end-organs [9-14].

Mexico currently disposes of therapeutic effective agents like monoclonal antibodies vs. PD-1 (nivolumab, pembrolizumab) and CTLA-4 (ipilimumab). In spite of the existence of immune un-blockers against PD-L1 ligand (atezolizumab, durvalumab, avelumab) in Mexico they are only available under investigation protocols. Besides this regulation pathway, the immune profile of an individual depends on multiple factors that include extrinsic circumstances such as the intestinal microbiome, the presence of infections, or exposure to environmental carcinogens, as well as the intrinsic properties of the tumour (genetic composition, cytokines secretion, etc.) [12-17]. In Mexico 
therapy use was initiated in 2012 with ipilimumab and in 2015 with nivolumab and pembrolizumab. Our institution (Médica Sur) was one of the pioneers in the utilisation of these drugs; thus, it is necessary to know the experience of Médica Sur Hospital to determine the clinical characteristics and adverse effects of patients who used some type of immunotherapy for distinct cancer types.

\section{Material and methods}

An observational, descriptive, cross-sectional study was performed on 70 patients of Médica Sur Hospital, who received immunotherapy based in ipilimumab, nivolumab, or pembrolizumab. The principal objective was to learn and determine the most frequent clinical and biochemical adverse effects in a period of 6-12 months. The secondary objective was to settle the response to treatment within the different cancer types. Descriptive statistics were used, involving central and dispersion tendency measures; range, average, median, mode, standard deviation, proportion, and percentages.

The inclusion criteria including having received treatment with immunotherapy for at least six months for metastatic oncologic disease (lung cancer, renal cancer, melanoma, colon cancer, and bladder cancer); all patients gave written, informed consent that was signed before immunotherapy treatment. Exclusion criteria included having incomplete follow-up by imaging studies and laboratory tests in our institution (Fig. 1).

\section{Results}

From a total of 105 patients who were eligible to receive immunotherapy for cancer (based on ipilimumab, nivolumab, and pembrolizumab), 70 (66.6\%) patients ful-

105 patients who met the inclusion criteria for initiation of immunotherapy based on ipilimumab, nivolumab, and pembrolizumab

35 excluded patients:

15 did not have complete clinical records

9 continued their follow-up in another hospital institution and did not complete a year of follow-up

7 did not report complete clinical characteristics and adverse effects

2 did not have complete follow-up with imaging studies to assess the progression

2 did not have complete follow-up with laboratory studies

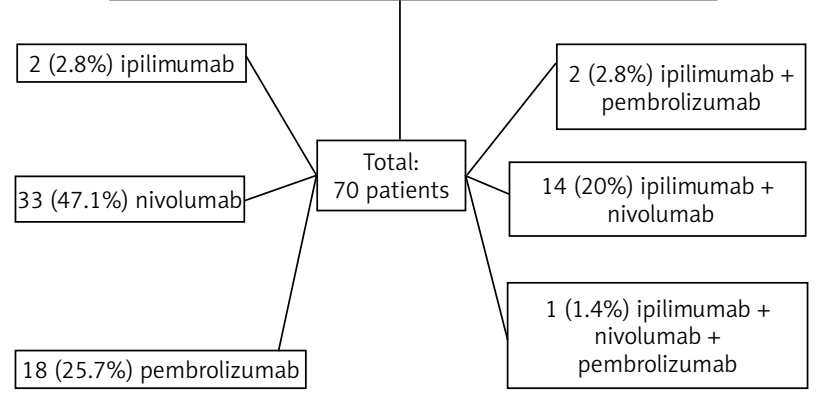

filled the inclusion criteria established for our study. Two of them $(2.8 \%)$ received monotherapy with ipilimumab, $33(47.1 \%)$ patients received single nivolumab monotherapy, $18(25.7 \%)$ were treated with pembrolizumab, two (2.8\%) received ipilimumab followed by pembrolizumab, $14(20 \%)$ ipilimumab + nivolumab, and one (1.4\%) received triple therapy with ipilimumab + nivolumab + pembrolizumab (Fig. 1). Nineteen patients $(27.1 \%)$ received associated chemotherapy with the immunotherapy.

\section{General characteristics}

From the 70 patients included in this study, 42 (60\%) were women with an average age of $60.73 \pm 13.64$ years (16-82 years). The average weight was $73.24 \pm 13.3 \mathrm{~kg}$ $(45-116 \mathrm{~kg})$. With respect to the Eastern Cooperative Oncology Group (ECOG) scale and validated by the World Health Organisation (WHO) to evaluate the quality of life of patients with cancer, 40 (57.1\%) presented an ECOG 0, 26 (37.1\%) ECOG 1, three (4.2\%) ECOG 2, one (1.4\%) ECOG 3, and no patient had ECOG 4.

Regarding to the presented comorbidities that patients had at the beginning of immunotherapy, it was found that 25 (35.7\%) did not have any, 28 (40\%) had high blood pressure, 18 (25.7\%) had type 2 diabetes mellitus, 13 (18.5\%) had dyslipidaemia, 3 (4.2\%) had ischaemic heart disease, eight (11.4\%) had hypothyroidism, eight (11.4\%) had chronic obstructive pulmonary disease, one (1.4\%) had epilepsy, four (5.7\%) had arrhythmias (auricular fibrillation, AV blocks), four (5.7\%) had asymptomatic hyperuricaemia, four $(5.7 \%)$ had benign prostatic hyperplasia, two $(2.8 \%)$ had stroke, and three (4.2\%) had venous thrombosis (PTE/ PTV). It is worth mentioning that only 16 patients (22.8\%) presented a single comorbidity, 12 (17.1\%) two comorbidities, 11 (15.7) three comorbidities, four (5.7\%) four comorbidities, and two (2.8\%) five comorbidities (Table 1 ).

The diseases that received immunotherapy included the following: melanoma - 17 (24.3\%), lung adenocarcinoma - 14 (20\%), small cell lung cancer - 8 (11.4\%), lung epidermoid carcinoma - 5 (7.1\%), mesothelioma - 5 (7.1\%), epidermoid cancer from the anal canal -3 (4.3\%), clear cell renal cancer - 2 (2.8\%), urothelial bladder carcinoma 2 (2.8\%), mucinous appendix adenocarcinoma - 2 (2.8\%), epidermoid bladder carcinoma - $1(1.4 \%)$, pelvic leiomyosarcoma - 1 (1.4\%), colon adenocarcinoma - 1 (1.4\%), gastric adenocarcinoma - 1 (1.4\%), gastroesophageal cancer - 1 (1-4\%), papillary thyroid cancer - 1 (1.4\%), renal adenocarcinoma + prostatic adenocarcinoma - 1 (1-4\%), melanoma + lung adenocarcinoma - 1 (1.4\%), gallbladder adenocarcinoma - 1 (1.4\%), melanoma + lung adenocarcinoma - 1 (1.4\%), high-grade osteosarcoma telangiectatic variety $-1(1.4 \%)$, cystic adenoid cancer -1 (1.4\%), epidermoid cervical cancer -1 (1.4\%), and ovarian adenocarcinoma -1 (1.4\%) (Table 2).

At the beginning of the diagnosis 66 (94.2\%) patients had metastatic disease being most frequently at ganglionar level 22 (31\%), lung - 14 (20\%), bone - 8 (11.4\%), liver $7(10 \%)$, and central nervous system $-5(7.1 \%)$. Of the $70 \mathrm{pa}$ tients, $45(64.3 \%)$ received surgical treatment associated

Fig. 1. Population in general with the exclusion criteria 
Table 1. General characteristics from the study sample

\begin{tabular}{|c|c|}
\hline Characteristics & $n(\%)$ \\
\hline Patients who fulfil inclusion criteria & $70(73)$ \\
\hline $\operatorname{Sex} M: F$ & $42(60): 28(40)$ \\
\hline Average age, mean (SD) & $60.73 \pm 13.64$ \\
\hline Average weight (kg) & $73.24 \pm 13.3$ \\
\hline Average of population with overweight (IMC) & $25.72 \pm 15.84$ \\
\hline \multicolumn{2}{|l|}{ Eastern Cooperative Oncology Group (ECOG) } \\
\hline ECOG 0 & $40(57.1)$ \\
\hline ECOG 1 & $26(37.1)$ \\
\hline ECOG 2 & $3(4.2)$ \\
\hline ECOG 3 & $1(1.4)$ \\
\hline ECOG 4 & 0 \\
\hline \multicolumn{2}{|l|}{ Comorbidities } \\
\hline None & $25(35.7)$ \\
\hline High blood pressure & $28(40)$ \\
\hline Type 2 diabetes mellitus & $18(25.7)$ \\
\hline Dyslipidaemia & $13(18.5)$ \\
\hline Ischaemic heart disease & $3(4.2)$ \\
\hline Hypothyroidism & $8(11.4)$ \\
\hline Chronic obstructive pulmonary disease & $8(11.4)$ \\
\hline Epilepsy & $1(1.4)$ \\
\hline Arrythmia (auricular fibrillation, AV blocks) & $4(5.7)$ \\
\hline Asymptomatic hyperuricaemia & $4(5.7)$ \\
\hline Benign prostatic hyperplasia & $4(5.7)$ \\
\hline Stroke & $2(2.8)$ \\
\hline $\begin{array}{l}\text { Venous thrombosis/pulmonary } \\
\text { thromboembolism (VT/PTE) }\end{array}$ & $3(4.2)$ \\
\hline \multicolumn{2}{|l|}{ Number of comorbidities presented by patients } \\
\hline 1 & $16(22.8)$ \\
\hline 2 & $11(17.1)$ \\
\hline 3 & $12(15.7)$ \\
\hline 4 & $4(5.7)$ \\
\hline 5 & $2(2.8)$ \\
\hline
\end{tabular}

with their baseline pathology, and 50 (71.4\%) subjects received some kind of chemotherapy before immunotherapy.

Concerning mutations, they were only performed in $21(30 \%)$ patients, revealing one $(1.4 \%)$ positive mutation for KRAS, three (4.2\%) positive for EGFR, three (4.2\%) positive for BRAF V600E, seven (10\%) negative for BRAF V600E, two $(2.9 \%)$ were negative for EGFR, ALK, and HER2 Neu, one $(1.4 \%)$ was negative for EGFR, two (2.9\%) were negative for BRAF, one (1.4\%) was ALK negative, and one (1.4\%) was negative for HER2 Neu. A positive PDL-1 was demonstrated in seven (10\%) patients.

Initially, before chemotherapy, the functional state ECOG classification was performed for a second time, reporting ECOG 0 - 17 (24.3\%), ECOG 1 - 42 (60\%), ECOG 2 6 (8.6\%), ECOG $3-3(4.3 \%)$, and ECOG $4-2$ (2.9\%). Within the executed studies, not only made for stratification and disease follow-up but also to corroborate the immunotherapy response to treatment, were the following: axial
Table 2. Pathologies that received immunotherapy

\begin{tabular}{lc} 
Pathology & $n(\%)$ \\
Melanoma & $17(24.3)$ \\
\hline Lung adenocarcinoma & $14(20)$ \\
Small cell lung cancer & $8(11.4)$ \\
Epidermoid lung carcinoma & $5(7.1)$ \\
Mesothelioma & $5(7.1)$ \\
Epidermoid anal cannel carcinoma & $3(4.3)$ \\
Clear cell renal cancer & $2(2.8)$ \\
Urothelial bladder carcinoma & $2(2.8)$ \\
Mucinous appendix carcinoma & $2(2.8)$ \\
Epidermoid bladder carcinoma & $1(1.4)$ \\
Pelvic leiomyosarcoma & $1(1.4)$ \\
Colon adenocarcinoma & $1(1.4)$ \\
Gastric adenocarcinoma & $1(1.4)$ \\
Gastroesophageal cancer & $1(1.4)$ \\
Papillary thyroid cancer & $1(1.4)$ \\
Renal adenocarcinoma + prostatic adenocarcinoma & $1(1.4)$ \\
Melanoma + lung adenocarcinoma & $1(1.4)$ \\
Gallbladder adenocarcinoma & $1(1.4)$ \\
High grade osteosarcoma telangiectasia variety & $1(1.4)$ \\
Cystic adenoid cancer & $1(1.4)$ \\
Epidermoid cervical cancer & $1(1.4)$ \\
Ovarian adenocarcinoma & $1(1.4)$
\end{tabular}

Table 3. Number of patients according to the applied immunotherapy

\begin{tabular}{lc} 
Immunotherapy & $n(\%)$ \\
Single immunotherapy & \\
\hline Ipilimumab & $2(2.8)$ \\
\hline Nivolumab & $33(47.1)$ \\
\hline Pembrolizumab & $18(25.7)$ \\
\hline Beginning therapy/following therapy & \\
\hline Ipilimumab/nivolumab & $14(20)$ \\
\hline Ipilimumab/pembrolizumab & $2(2.8)$ \\
\hline Ipilimumab, nivolumab and pembrolizumab & $1(1.4)$ \\
\hline $\begin{array}{l}\text { Chemotherapy or radiotherapy associated with } \\
\text { immunotherapy }\end{array}$ & $19(27.1)$ \\
\hline Bevacizumab & $4(5.7)$ \\
\hline Radiotherapy & $2(2.8)$ \\
\hline Paclitaxel + cisplatin + bevacizumab & $2(2.8)$ \\
\hline Capecitabine & $2(2.8)$ \\
\hline Etoposide + carboplatin + denosumab & $1(1.4)$ \\
\hline Pemetrexed + oxaliplatin + bevacizumab & $1(1.4)$ \\
\hline Vemurafenib + dabrafenib + trametinib & $1(1.4)$ \\
\hline Interferon & $1(1.4)$ \\
\hline Carboplatin + dacarbazine & $1(1.4)$ \\
\hline Pemetrexed + carboplatin & $1(1.4)$ \\
\hline
\end{tabular}


computed tomography - 46 (65.7\%), positron emission tomography - 42 (60\%), magnetic resonance - 9 (12.9\%), colonoscopy -3 (4.3\%).

As mentioned before, two (2.8\%) patients received monotherapy with ipilimumab, 33 (47.1\%) with nivolumab, and 18 (25.7\%) with pembrolizumab. Combined therapy was applied in 14 (20\%) patients with ipilimumab + nivolumab, from which 10 were applied concomitantly and four in a sequential way, two (2.8\%) received ipilimumab + pembrolizumab in sequence (first received ipilimumab for melanoma and further nivolumab + pembrolizumab were added in sequence). Nineteen (27.1\%) received associated chemotherapy or radiotherapy in spite of immunotherapy: bevacizumab - four (5.7\%), radiotherapy - two (2.8\%), placlitaxel + cisplatin + bevacizumab - two (2.8\%), capecitabine - two (2.8\%), etoposide + carboplatin + denosumab - one (1.4\%), pemetrexed + oxaliplatin + bevacizumab -

Table 4. Adverse effects

\begin{tabular}{|c|c|}
\hline Adverse effects & $n(\%)$ \\
\hline \multicolumn{2}{|l|}{ General clinical adverse effects } \\
\hline Fatigue & $32(45.7)$ \\
\hline Asthenia & $30(42)$ \\
\hline Adynamia & $28(40)$ \\
\hline Fever & $2(2.9)$ \\
\hline \multicolumn{2}{|l|}{ Gastrointestinal adverse effects } \\
\hline Nausea & $8(11.4)$ \\
\hline Vomiting & $4(5.7)$ \\
\hline Diarrhoea & $8(11.4)$ \\
\hline Hyporexia & $8(11.4)$ \\
\hline Pancreatitis & $1(1.4)$ \\
\hline Haemorrhagic colitis & $1(1.4)$ \\
\hline \multicolumn{2}{|l|}{ Respiratory adverse effects } \\
\hline Pneumonitis & $10(14.28)$ \\
\hline Allergic rhinitis & $1(1.4)$ \\
\hline \multicolumn{2}{|l|}{ Muscular adverse effects } \\
\hline Myalgias & $1(1.4)$ \\
\hline \multicolumn{2}{|l|}{ Endocrinological adverse effects } \\
\hline Hypothyroidism & $4(5.71)$ \\
\hline Hyperglycaemia & $1(1.4)$ \\
\hline Hypophysitis & $2(2.9)$ \\
\hline \multicolumn{2}{|l|}{ Skin adverse effects } \\
\hline Rash & $7(10)$ \\
\hline Pruritus & $5(7.14)$ \\
\hline Vitiligo & $2(2.9)$ \\
\hline Alopecia & $1(1.4)$ \\
\hline \multicolumn{2}{|l|}{ Laboratory adverse effects } \\
\hline TSH elevation & $2(2.9)$ \\
\hline Glucose elevation & $1(1.4)$ \\
\hline Lipase/amylase elevation & $1(1.4)$ \\
\hline ALT and AST increase & $3(4.28)$ \\
\hline DHL above normal upper limits & $10(14.28)$ \\
\hline
\end{tabular}

one $(1.4 \%)$, vemurafenib + dabrafenib + trametinib - one (1.4\%), interferon - one (1.4\%), carboplatin + dacarbazine - one (1.4\%), and pemetrexed + carboplatin - one (1.4\%) (Table 3).

\section{Complications and adverse effects}

Fifteen (21.4\%) patients presented central nervous system metastasis, from which only one presented it during immunotherapy treatment. Within the manifested complications, the following were presented: cardiac tamponade - two (2.9\%), intestinal occlusion - one (1.4\%), and haemoptysis - one (1.4\%). The clinical and biochemical adverse effects (Table 4) included general effects: fatigue - 32 (42.7\%), asthaenia - 30 (42\%), adynamia - 28 (40\%); gastrointestinal: nausea - eight (11.4\%), vomiting - four (5.7\%), diarrhoea - eight (11.4\%), hyperoxia - eight (11.4\%), pancreatitis - one (1.4\%), haemorrhagic colitis - one (1.4\%); respiratory: pneumonitis - 10 (14.28\%), allergic rhinitis - one (1.9\%); muscular: myalgias - one (1.4\%), endocrinology: hypothyroidism - four (5.71\%), hyperglycaemia - one (1.4\%), hypophysitis - two (2.9\%); skin: rash - seven (10\%), pruritus - five (7.14\%), vitiligo - two (2.9\%), alopecia - one (1.4\%); laboratory: TSH elevation - two (2.9\%), glucose elevation - one (1.4\%), lipase/amylase elevation - one (1.4\%), ALT elevation - three (4.28\%), AST elevation - three (4.28\%), and DHL above superior normal limit 10 (14.28\%). The mean number of days of hospitalisation was $1.27 \pm 4.48$ (0-31 days).

\section{Response to treatment}

Speaking of the response to treatment, complete response was presented in seven (10\%) patients, from which three $(4.28 \%)$ were treated with nivolumab, two (2.96\%) with pembrolizumab, and two (2.96\%) with ipilimumab + nivolumab. Partial response was presented in 11 (15.71\%), from which four (5.7\%) were treated with nivolumab, one (1.4\%) with ipilimumab, one (1.4\%) with pembrolizumab, and five $(7.14 \%)$ with ipilimumab + nivolumab. The disease remained stable in 33 (47.14\%), from which one (1.4\%) was treated with ipilimumab, 18 (25.71\%) with nivolumab, eight (11.4\%) with pembrolizumab, and five (7.14\%) with ipilimumab + nivolumab. Disease progression was observed in 19 (27.1\%); eight patients (11.4\%) were treated with nivolumab. Two of them (2.9\%) died, one of them had as baseline pathology stage IV lung adenocarcinoma, ECOG 2 , and the other one stage IV epithelioid mesothelioma, ECOG 4. Seven patients (10\%) progressed with pembrolizumab, and one of them (1.4\%) died with stage IV lung adenocarcinoma as baseline pathology, ECOG 3, three (4.2\%) with ipilimumab + nivolumab, and one (1.4\%) with ipilimumab + pembrolizumab, who died with stage IV malignant melanoma, ECOG 2 (Table 5).

\section{Discussion}

Since the beginning, two completely human IgG monoclonal antibodies: anti-CTLA-4 - ipilimumab (MDX-010) and tremelimumab (CP-675 206) have been proven as monotherapy also in combination in clinical trials phase II and phase III, since 2001 and 2002 respectively, and it was 
highlighted that the pattern and duration of the immune response associated with these new modalities differ from those related with cytokines and cytotoxic agents [18, 19].

The earliest positive results with immune checkpoint inhibitors (ICls) in randomised trials were obtained for metastatic melanoma with ipilimumab; a 2010 published essay demonstrated superiority of ipilimumab as second-line treatment against the comparison pattern based in the gp100 vaccine (average survival of 10.1 and 6.4 months, respectively), which permitted its rapid approbation for the regulatory agencies in the US. The approved dosage is $3 \mathrm{mg} / \mathrm{kg}$ every three weeks, to complete four applications [20].

The introduction of PD-1 antibodies has its beginning in the evaluation of antitumour activity and the safety of BMS-936558, a specific PD-1 antibody that blocks at a dosage of $0.1-10 \mathrm{mg} / \mathrm{kg}$ every two weeks, evaluating the response every eight weeks for the treatment sequence cycle, which produced objective responses in approximately one in every four to one in five patients with small-cell lung cancer, melanoma, or renal cell cancer; the adverse effects profile does not seem to exclude its use [10].

The effectiveness and activity of antibody against PD-L1 (BMS-936559) was validated from a phase I multicentric trial in which durable tumour regression was reported (objective response rate of 6 to 17\%) and prolonged stability of the disease (rate from $12 \%$ to $41 \%$ at 24 weeks) in patients with advanced cancer, including non-small cell lung cancer, melanoma, and renal cell cancer [21].

By virtue of the great reported global survival of metastatic melanoma, the FDA approved the monoclonal antibody vs. CTLA-4 (ipilimumab) in March of 2011, and then approved the humanised monoclonal antibody against PD-1 (pembrolizumab) and PD-1 (nivolumab) in September and December of 2014, respectively [20, 22-24]. A year later the FDA approved the combination (ipilimumab + nivolumab) in a phase III trial (CheckMate 067) for presentation of free survival progression evidence in metastatic melanoma [25-27], and utility of ipilimumab as surgically resectable melanoma treatment was restated [28]. In 2017 (KEYNOTE-006) the multicentric, randomised phase III trial assigned patients in a $1: 1: 1$ ratio (pembrolizum$a b$ every two and three weeks or ipilimumab every three weeks) demonstrating that pembrolizumab maintained its superiority in relation to disease-free and global survival of $28-31 \%$ to 24 months vs. $14 \%$ with ipilimumab [29]. It should be noted that in the KEYNOTE-001 trial the optimal treatment duration controversy emerges as with a short pembrolizumab treatment it was observed a prolonged complete response after suspension of the treatment [30]. Also, a combination at standard dose of ipilimumab + pembrolizumab was applied in low dosage in a phase I trial (KEYNOTE 0-29), in which increased rates of response and survival improvement were highlighted as being better than with monotherapy, whereby higher rates of toxicity were presented [31].

In 2015 immunotherapy gained great importance in lung cancer due to the approbation of nivolumab in the CheckMate 017 trial, not only for presenting global survival evidence of 9.2 months and best response up to $20 \%$, but
Table 5. Response to treatment according to applied immunotherapy

\begin{tabular}{|c|c|}
\hline Response to treatment & $n(\%)$ \\
\hline Complete response & $7(10)$ \\
\hline Nivolumab & $3(4.28)$ \\
\hline Pembrolizumab & $2(2.96)$ \\
\hline Nivolumab + ipilimumab & $2(2.96)$ \\
\hline Partial response & $11(15.71)$ \\
\hline Nivolumab & $4(5.7)$ \\
\hline Ipilimumab & $1(1.4)$ \\
\hline Pembrolizumab & $1(1.4)$ \\
\hline Nivolumab + ipilimumab & $5(7.14)$ \\
\hline Stable disease & $33(47.14)$ \\
\hline Ipilimumab & $1(1.4)$ \\
\hline Nivolumab & $18(25.71)$ \\
\hline Pembrolizumab & $8(11.4)$ \\
\hline Nivolumab + ipilimumab & $5(7.14)$ \\
\hline Progression of disease & $19(27.1)$ \\
\hline Nivolumab* & $8(11.4)^{*}$ \\
\hline Pembrolizumab* & $7(10)^{* *}$ \\
\hline Nivolumab + ipilimumab & $3(4.2)$ \\
\hline Ipilimumab + pembrolizumab & $1(1.4)^{\star \star \star}$ \\
\hline
\end{tabular}

two of them (2.9\%) deceased, one of them had as baseline pathology stage IV lung adenocarcinoma, ECOG 2, and the other one stage IV epithelioid mesothelioma, ECOG 4, ${ }^{*}$ one of them (1.4\%) deceased with stage IV, lung adenocarcinoma as baseline pathology, ECOG 3, * deceased for stage IV malignant melanoma, ECOG 2

also progression-free survival of 3.5 months and lower adverse effect rates of $7 \%$, in comparison with docetaxel as a second-line treatment for non-small cell squamous lung cancer [32]. In the same year, efficacy was proven as a second-line treatment in non-microcytic lung cancer stage IV (non-squamous, adenocarcinoma) over nivolumab, improved response rate of $19 \%$, with a higher global survival of 12.2 months and fewer adverse effects (10\%) in comparison with docetaxel in the CheckMate 057 trial [33].

Pembrolizumab also achieves great results in global survival of up to 10.4-12.7 months and fewer adverse effects, at $13-16 \%$, in comparison with docetaxel as second-line treatment for non-small cell stage IV lung cancer with positive PD-L1 in < 50\% in the KEYNOTE-010 trial [34]. The last OAK multicentric, randomised, controlled, phase III trial demonstrated improvement in the progression-free survival as globally as with the response rate and less adverse effects with atezolizumab in comparison to docetaxel as second-line treatment in non-small cell lung cancer [35].

In a phase I trial nivolumab at dose of $3 \mathrm{mg} / \mathrm{kg}$ every two weeks demonstrated an objective response of $23 \%$ and $28 \%$ in patients who presented PD-L1 biomarker, with an average global survival of 19.4 months and $19 \%$ of serious effects in non-small cell lung cancer [36].

In the KEYNOTE-024 trial, pembrolizumab vs. chemotherapy based on platins for microcytic lung cancer with PD-L1 expression in more than $50 \%$ of the tumour cells, it was shown than pembrolizumab had a better response 
rate in $44.8 \%$, higher progression-free survival of 10.3 months, global survival of 80.2 months, and fewer adverse effects in $26.6 \%$, a pattern that led to incorporating pembrolizumab in the NCCN guides (Version 3.2017) as firstline treatment in patients who fulfil those criteria [37]. In another randomised trial, CheckMate 026, nivolumab was compared with standard platin chemotherapy in non-microcytic lung cancer with PD-L1 expression of at least 1\%. As first-line treatment, there was no meaningful superiority for nivolumab and just fewer adverse effects were corroborated as serious 18\% [38].

Likewise, there are two assays in which combination of immunotherapy and chemotherapy is valuated: CheckMate 012 (nivolumab at different dosage + chemotherapy based in platins), in which objective responses are observed in $47 \%$ and a global two-year survival of $62 \%$ [39]. In another trial (KEYNOTE 021) pembrolizumab + QT (carboplatin + pemetrexed) was compared with the same pattern of isolated QT; both arms further received maintenance pemetrexed. The response rates were favourable in $55 \%$ due to combination and with similar frequency of serious adverse effects [40].

In a phase III trial that combined platins + etoposide and ipilimumab for microcytic stage IV lung cancer, a discrete improvement was shown in disease-free survival; however, the said combination did not overcome the pattern of isolated chemotherapy [41]. At the end of 2016 CheckMate 032 a comparison was performed between ipilimumab + nivolumab vs. single nivolumab, denoting that the combination of both immunotherapies gave better objective responses in 10-19\% with acceptable toxicity in patients with microcytic lung cancer recurrence within six months after first treatment, a pattern which marked the incorporation of this treatment to the NCCN (Version 3.2017) [42].

Recently, in 2015 November, the FDA approved nivolumab for presenting evidence of global survival in renal cell carcinoma as second-line treatment compared to everolimus [43]. Also, promising results were obtained with nivolumab in a phase I trial [44] that included 23 patients with refractory or recurrent Hodgkin's classic lymphoma. An objective response rate of $87 \%$ was obtained and none of the patients showed disease progression during nivolumab therapy. Pembrolizumab is active in other solid tumours, such as lung, head, neck, triple-negative breast cancer, and renal cell carcinoma [25].

Immune checkpoint inhibitors (ICls) were included in clinical practice as a second-line option after an initial chemotherapy regimen, and in the last year positive results have been reported from randomised trials in which they were compared in the first line with standard chemotherapy. Responses have been surprising and durable, but less than 20-25\% in unselected patients, so it is essential that factors predicting efficacy be identified. One such biomarker is PD-L1, but the different methods used to detect it have produced mixed results [45].

The precise pathophysiology of $\mathrm{ICl}$-mediated immune-related adverse effects (irAES) is currently unknown. Some research shows that irAES may result from some combination of autoreactive T cells, autoantibodies, and/ or proinflammatory cytokines. The adverse effects are no-
irAES or irAES. The early- and later-onset irAES may result from distinct mechanisms that have yet to be elucidated. Typical earlier-onset, common irAES appears to involve generalised epithelial inflammation and may be observed in the form of rash, colitis, and pneumonitis. Later-onset irAES, which is typically less common, can include neurologic events and hypophysitis, among others [46].

In our study, any degree of irAES was generally reported in 50 (71.4\%) patients, in 10 (14.2\%) of whom it was high grade. The reported incidence of any-grade irAES associated with single-agent $\mathrm{ICI}$ treatment ranges widely across agents and trials, from approximately 15 to $90 \%$. With anti-CTLA-4 antibodies (ipilimumab), the overall incidence of any-grade irAES is $72 \%$, and $24 \%$ for high-grade irAES $[20,47]$.

A randomised, double-blind, phase III trial in patients with unresectable or metastatic melanoma revealed a dose-dependent effect in treatment-related AEs for patients receiving ipilimumab at a dose of $3 \mathrm{mg} / \mathrm{kg}(n=362)$ or $10 \mathrm{mg} / \mathrm{kg}(n=364)$. The most common irAES was rash (13-15\%), pruritus (22\%), diarrhoea (17-27\%), and fatigue (10\%). High-grade irAES was reported in $18 \%$ and $30 \%$ of the $3 \mathrm{mg} / \mathrm{kg}$ and $10 \mathrm{mg} / \mathrm{kg}$ treatment groups, respectively. The most common high-grade AEs, including diarrhoea (6-10\%), colitis (2-5\%), elevated liver enzymes (2\%), and hypophysitis (2\%), were all more common at the higher dose of ipilimumab [48]. We reported rash (10\%) and pruritus (7.1\%) much less frequently than that reported in the literature. Our results are similar to this study with respect to grade 3 irAES except for colitis (1.4\%) and diarrhoea (2.9\%), which occurred less frequently.

The incidence of any-grade irAES associated with anti-PD-1/PD-L1 bodies was reported in 27-30\%, and in 5-8\% for high-grade irAES. The most commonly observed AES were dermatology (vitiligo in relation with melanoma) and gastrointestinal (colitis), followed by endocrine (hypothyroidism, hepatic (elevated liver enzymes), and pneumonitis (5-6.7\%) events [46-49].

De Velasco et al. recently reported on the incidence of the most common immune checkpoint inhibitor (ICI)-associated irAES in a meta-analysis of 21 randomised phase II/III trials conducted from 1996 to 2016, which included a total of 6528 patients who received monotherapy (atezolizumab, $n=751$; ipilimumab, $n=721$; nivolumab, $n=1534$; pembrolizumab, $n=1522$ ) and 4926 patients in placebo or standard therapy control arms using chemotherapy or biologic agents. When compared to patients in the trial control arms, patients receiving $\mathrm{ICl}$ s were found to be at greater risk for any-grade immune-related colitis, AST elevation, rash, hypothyroidism, and pneumonitis. Within this cohort, across all $\mathrm{ICls}$, the incidence of grade $3 / 4$ events was $1.5 \%$ for colitis, $1.5 \%$ for liver toxicity, $1.1 \%$ for rash, $0.3 \%$ for hypothyroidism, and $1.1 \%$ for pneumonitis. High-grade colitis and rash were significantly more common among patients on ipilimumab than in those receiving PD-1/PD-L1 inhibitor [50].

A 2018 meta-analysis compared the data on toxicity profiles of PD-1 and PD-L1 inhibitors from 23 studies that occurred between 2013 and 2016 (PD-1, $n=3284$; PD-L1, $n=2460$ ). A near-significant trend revealed irAES to be more common with PD-1 vs. PD-L1 blockade (16\% vs. $11 \%$; $p=0.07)$. However, the incidence of severe irAES was not 
significantly different between PD-L1 and PD-1 inhibitors, ( $5 \%$ vs. $3 \%, p=0.4)$. Pneumonitis occurred twice as often with PD-1 inhibitors ( $4 \%$ vs. $2 \% ; p=0.01$ ), and hypothyroidism was also more common with PD-1 inhibitors (6.7\% vs. $4.2 \% ; p=0.07$ ) [51]

The combination of CTLA-4 and PD-1/PD-L1 blockade has only been approved for patients with metastatic melanoma. Treatment-related irAES were observed in 95\% of patients. In $55 \%$ of patients these irAES were of grade 3 or higher [49].

It is worth mentioning that the most frequent adverse effects were the general symptoms (asthaenia, fatigue, adynamia, gastrointestinal [nausea, diarrhoea, and hyporexia] and skin manifestations such as rash and pruritus); however, the adverse effects with major impact in tolerance, follow-up, hospital stay, and mortality related to immunotherapy were pneumonitis, hypothyroidism, hypophysitis, type 1 diabetes, and haemorrhagic colitis. We observed an incidence of pneumonitis in our population almost 2-4 times higher than that in previously published reports in a Caucasian population (pneumonitis 2.6 to $6.7 \%)$ [49]. Three cases (4.28\%) were classified as highgrade pneumonitis (grade 3 ), and only two (2.85\%) patients had received chest radiotherapy before and during immunotherapy. One of the probable causes of pneumonitis in this group of patients was the presence of lung damage related to tumour burden, exposure to smoking, and the presence of chronic obstructive pulmonary disease or fibrosis.

Pneumonitis associated with checkpoint blockade is a toxicity of variable onset and clinical, radiological, and pathological appearance, which has been observed with anti-PD-1/PD-L1 monoclonal antibodies (MoAbs) and, more rarely, with anti-CTLA-4 MoAbs. It is more common when anti-PD-1/PD-L1 MoAbs are combined with anti-CTLA-4 MoAbs. Several cases of distinct life-threatening respiratory events have been reported under anti-CTLA-4 monotherapy. Acute interstitial pneumonitis/diffuse alveolar damage syndrome (DADS) is the most acute, life-threatening event. Patients with a history of autoimmune disease or who are being actively treated for an autoimmune disease are at risk for worsening of their autoimmune disease while on immune checkpoint blockade. Similarly, patients that have had irAES on ipilimumab are at risk of developing irAES following anti-PD-1 treatment and vice versa. Results from these retrospective series showed a higher rate of grade 3 to 4 toxicity in patients treated with ipilimumab following anti-PD-1 (up to 35\%), and patients with grade 3 to 4 toxicity on ipilimumab followed by anti-PD-1 developed grade 3 to 4 irAES in $>20 \%$ of cases. The pneumonitis associated with radiotherapy is very frequent, so it is also important to avoid the combination of immunotherapy and radiotherapy, especially mediastinum [47, 50, 51].

It is remarkable that laboratory-reported alterations allowed us to come after the pattern with follow-up studies of thyroid profile to discard hypothyroidism, fast glucose levels to discard type 2 diabetes mellitus, liver function proofs to monitor the values or increases of liver enzymes as ALT and AST, as well as lipase/amylase to evaluate pan- creatitis risk, and also imaging studies like cranial TAC/ MRI to discard hypophysitis, X-ray/thoracic TAC to discard pneumonitis, and colonoscopy in suspicion of haemorrhagic colitis; all of these correlated with clinical patient symptoms.

\section{Conclusions}

Immunotherapy based in ipilimumab, nivolumab, and pembrolizumab is a better combined therapy either with surgery or with chemotherapy in patients with metastatic diseases in clinical stage IV, with recurrence or bad response to baseline treatments in different types of cancer, mostly in melanoma and lung cancer. The most frequent adverse effects generally do not conditionate the suspension of treatment nor hospital-stay prolongation; however, there are some adverse effects that actually have an impact in evolution, hospital-stay, and mortality, such as pneumonitis, hypophysitis, type 1 diabetes mellitus, and haemorrhagic colitis. Hence, it is imperative to obtain a precise image and perform routine biochemical follow-up.

\section{The authors declare no conflict of interest.}

\section{References}

1. National Cancer Institute. NCl dictionary of cancer terms: immunotherapy. http://www.cancer.gov/dictionary?print $=1 \&$ cdrid $=45729$ (access: 12.09.2017).

2. Schreiber RD, Old LJ, Smyth MJ. Cancer immunoediting: integrating immunity's roles in cancer suppression and promotion. Science 2011; 331: 1565-1570.

3. Chen DS, Mellman I. Elements of cancer immunity and the cancer-immune setpoint. Nature 2017; 541: 321-330.

4. Burugu S, Dancsok AR, Nielsen TO. Emerging targets in cancer immunotherapy. Semin Cancer Biol 2018; 52: 39-52.

5. Pierard GE, Aubin F, Humbert P. Ipilimumab, a promising immunotherapy with increased overall survival in metastatic melanoma? Dermatol Res Pract 2012; 2012: 182157.

6. Liang SC, Latchman YE, Buhlmann JE, Tomczak MF, Horwitz BH, Freeman GJ, Sharpe AH. Regulation of PD-1, PD-L1, and PD-L2 expression during normal and autoimmune responses. Eur J Immunol 2003; 33: 2706-2716.

7. Zhang X, Schwartz JC, Guo X, et al. Structural and functional analysis of the costimulatory receptor programmed death-1. Immunity 2004; 20: 337-347.

8. Topalian SL, Hodi FS, Brahmer JS, et al. Safety, activity and immune correlates of anti-PD-1 antibody in cancer. N Engl J Med 2012; 366: 2443-2454.

9. Loke P, Allison JP. PD-L1 and PD-L2 are differentially regulated by Th1 and Th2 cells. Proc Natl Acad Sci USA 2003; 100: 5336-5341.

10. Yamazaki T, Akiba $H$, Iwai $H$, et al. Expression of programmed death 1 ligands by murine T cells and APC. J Immunol 2002; 169: 5538-5545.

11. Brown JA, Dorfman DM, Ma FR, Sullivan EL, Munoz O, Wood CR, Greenfield EA, Freeman GJ. Blockade of programmed death-1 ligands on dendritic cells enhances T cell activation and cytokine production. J Immunol 2003; 170: 1257-1266.

12. Pardoll DM. The blockade of immune checkpoints in cancer immunotherapy. Nat Rev Cancer 2012; 12: 252-264.

13. Mellman I, Coukos G, Dranoff G. Cancer immunotherapy comes of age. Nature 2011; 480: 480-489.

14. Motz GT, Coukos G. Deciphering and reversing tumor immune suppression. Immunity 2013; 39: 61-73.

15. Ichim CV. Revisiting immunosurveillance and immunostimulation: Implications for cancer immunotherapy. J Transl Med 2005; 3: 8. 
16. Vesely MD, Kershaw MH, Schreiber RD, Smyth MJ. Natural innate and adaptive immunity to cancer. Annu Rev Immunol 2011; 29: 235-271.

17. Schreiber RD, Old LJ, Smyth MJ. Cancer immunoediting: integrating immunity's roles in cancer suppression and promotion. Science 2011; 331: 1565-1570.

18. Eggermont AM, Schadendorf D. Melanoma and immunotherapy. Hematol Oncol Clin N Am 2009; 23: 547-564.

19. Mansh M. Ipilimumab and Cancer Immunotherapy: A new Hope for Advanced Stage Melanoma. Yale J Biol Med 2011; 84: 381-389.

20. Hodi FS, O’Day SJ, McDermott DF, et al. Improved survival with Ipilimumab in patients with metastatic melanoma. N Engl J Med 2010; 363: 711-723.

21. Brahmer JR, Tykodi SS, Chow LQ, et al. Safety and activity of anti-PD-L1 antibody in patients with advanced cancer. New Eng J Med 2012; 366: 2455-2465.

22. Ribas A, Puzanov I, Dummer R, et al. Pembrolizumab versus inves tigator-choice chemotherapy for ipilimumab-refractory melanoma (KEYNOTE-002): a randomised, controlled,phase 2 trial. Lancet Oncol 2015; 16: 908-918.

23. Weber JS, D’Angelo SP, Minor D, et al. Nivolumab versus chemotherapy in patients with advanced melanoma who progressed after anti-CTLA-4 treatment (CheckMate 037): A randomized, controlled, open-label, phase 3 trial. Lancet Oncol 2015; 16: 375-384.

24. Robert C, Thomas L, Bondarenko I, et al. I pilimumab plus dacarbazine for previously untreated metastatic melanoma. N Engl J Med 2011; 364: 2517-2526.

25. Postow MA, Chesney J, Pavlick AC, et al. Nivolumab and ipilimumab versus ipilimumab in untreated melanoma. N Engl J Med 2015; 372: 2006-2017.

26. Larkin J, Hodi FS, Wolchok JD. Combined nivolumab and ipilimumab or monotherapy in untreated melanoma. N Engl J Med 2015; 373: 1270-1271.

27. Wolchok JD, Kluger H, Callahan MK, et al. Nivolumab plus ipilim umab in advanced melanoma. N Engl J Med 2013; 369: 122-133.

28. Eggermont AM, Chiarion-Sileni V, Grob JJ, et al. Adjuvant ipilimumab versus placebo after complete resection of high-risk stage III melanoma (EORTC 18071): a randomized, double-blind, phase 3 trial. Lancet Oncol 2015; 16: 522-530.

29. Robert C, Schachter J, Long GV, et al. Pembrolizumab versus I pilimumab in Advanced Melanoma. N Engl J Med 2015; 25: 2521-2532.

30. Ramalingam S, Hui R, Gandhi L, Carcereny E, Felip E, Ahn MJ. P2.39: Long-Term OS for Patients With Advanced NSCLC Enrolled in the KEYNOTE-001 Study of Pembrolizumab. J Thorac Oncol 2016; 11 (Suppl): S241-S242.

31. Long GV, Atkinson V, Cebon IS, et al. Standard-dose pembrolizum$a b$ in combination with reduced-dose ipilimumab for patients with advanced melanoma (KEYNOTE-029): an open-label, phase 1b trial. Lancet Oncol 2017; 18: 1202-1210.

32. Brahmer JR, Tykodi SS, Chow LQ, et al. Safety and activity of anti-PD-L1 antibody in patients with advanced cancer. N Engl J Med 2012; 366: 2455-2465.

33. Borghaei H, Paz-Ares L, Horn L, et al. Nivolumab versus docetaxel in advanced nonsquamous non-small-cell lung cancer. N Engl J Med 2015; 373: 1627-1639.

34. Herbst RS, Baas P, Kim DW, et al. Pembrolizumab versus docetaxel for previously treated, PD-L1-positive, advanced non-small-cell lung cancer (KEYNOTE-010): a randomized controlled trial. Lancet 2016; 387: 1540-1550

35. Rittmeier A, Barlesi F, Waterkamp D, et al. Atezolizumab versus docetaxel in patients with previously treated non-small cell lung cancer (OAK): A phase 3, open-label, multicentre randomized controlled trial. Lancet 2017; 389: 255-265.

36. Gettinger S, Rizvi NA, Chow LQ, et al. Nivolumab monotherapy for first line treatment of advanced non-small cell lung cancer. J Clin Oncol 2016; 34: 2980-2987.

37. Reck M, Rodriguez-Abreu D, Robinson AG, et al. Pembrolizumab versus chemotherapy for PD-L1 positive non-small cell lung cancer. N Engl J Med 2016; 375: 1823-1833.

38. Carbone DP, Reck M, Paz-Ares L, et al. CheckMate 026 Investigators. First Line Nivolumab in Stage IV or Recurrent Non-Small-Cell Lung Cancer. N Engl J Med 2017; 376: 2415-2426.
39. Rizvi NA, Hellmann MD, Brahmer J, et al. Nivolumab in combination with platinum-based doublet chemotherapy for first line treatment of advanced non-small cell lung cancer. J Clin Oncol 2016; 34: 2969-2979.

40. Langer CJ, Gadgeel SM, Borghaei H, Papadimitrakopoulou VA Patnaik A, Powell SF. Carboplatin and pemetrexed with or without pembrolizumab for advanced, non-squamous non-small-cell lung cancer: A randomized, phase 2 cohort of the open-label KEYNOTE-021 study. Lancet Oncol 2016; 17: 1497-1508.

41. Reck M, Luft A, Szczesna A, et al. Phase III randomized trial of ipililumab plus etoposide and platinum versus placebo plus etoposide and platinum in extensive stage small cell lung cancer. J Clin Oncol 2016; 34: 3740-3748.

42. Antonia SJ, López Martín JA, Bendell J, et al. Nivolumab alone and nivolumab plus ipilimumab in recurrent small cell lung cancer (CheckMate 032): A multicentre open-label phase 1/2 trial. Lancet Oncol 2016; 17: 883-895.

43. Motzer RJ, Escudier B, McDermott DF, et al. Nivolumab versus everolimus in advanced renal-cell carcinoma. N Engl J Med 2015; 373 : 1803-1813.

44. Ansell SM, Lesokhin AM, Borrello I, et al. PD-1 blockade with nivolumab in relapsed or refractory Hodgkin's lymphoma. N Engl J Med 2015; 372: 311-319.

45. Sánchez de Cos Escuín J. New Immunotherapy and Lung Cancer. Arch Bronconeumol 2017; 53: 682-687.

46. National Comprehensive Cancer Network. National Comprehensive Cancer Network clinical practice guidelines in oncology. Management of immunotherapy-related toxicities. Version 1.2019 https://www.nccn.org/professionals/physician_gls/pdf/immunotherapy.pdf (access: 30.03.2019)

47. Haanen JBAG, Carbonnel F, Robert C, Kerr KM, Peters S, Larkin J, Jordan K; ESMO Guidelines Committee. Management of toxicities from immunotherapy: ESMO Clinical Practice Guidelines for diagnosis, treatment and follow-up. Ann Oncol 2017; 28 (Suppl 4): iv119-iv142.

48. Ascierto PA, Del Vecchio M, Robert C, et al. Ipilimumab 10 mg/kg versus ipilimumab $3 \mathrm{mg} / \mathrm{kg}$ in patients with unresectable or metastatic melanoma: a randomised, double-blind, multicentre, phase 3 trial. Lancet Oncol 2017; 18: 611-622.

49. Khunger M, Rakshit S, Pasupuleti V, Hernandez AV, Mazzone P, Stevenson J, Pennell NA, Velcheti V. Incidence of Pneumonitis With Use of Programmed Death 1 and Programmed Death-Ligand $1 \mathrm{In}$ hibitors in Non-Small Cell Lung Cancer: A Systematic Review and Meta-Analysis of Trials. Chest 2017; 152: 271-281.

50. De Velasco G, Je Y, Bosse D, et al. Comprehensive Meta-analysis of Key Immune-Related Adverse Events from CTLA-4 and PD-1/ PD-L1 Inhibitors in Cancer Patients. Cancer Immunol Res 2017; 5 : 312-318.

51. Pillai RN, Behera M, Owonikoko TK, et al. Comparison of the toxicity profile of PD-1 versus PD-L1 inhibitors in non-small cell lung cancer: A systematic analysis of the literature. Cancer 2018; 124: 271-277.

\section{Address for correspondence}

\section{María Eugenia Torres-Pérez}

Internal Medicine Department

Médica Sur Hospital

Puente de Piedra \#150

Colonia Toriello Guerra, Delegación Tlalpan

México City, México

e-mail: dra.marusatorres@hotmail.com

Submitted: 30.05 .2019

Accepted: 15.08.2019 